\title{
Politique
}

\section{Répertoire des thèses et mémoires en science politique} 1987-1988

Numéro 14, automne 1988

Sport et politique et Le NPD

URI : https://id.erudit.org/iderudit/040615ar

DOI : https://doi.org/10.7202/040615ar

Aller au sommaire du numéro

Éditeur(s)

Société québécoise de science politique

ISSN

0711-608X (imprimé)

1918-6584 (numérique)

Découvrir la revue

Citer ce document

(1988). Répertoire des thèses et mémoires en science politique 1987-1988.

Politique, (14), 205-210. https://doi.org/10.7202/040615ar d'utilisation que vous pouvez consulter en ligne.

https://apropos.erudit.org/fr/usagers/politique-dutilisation/ 


\section{Répertoire des thèses et mémoires en science politique 1987-1988}

\section{Université Laval}

\section{Thèses}

Diop, Abdoulaye

Mukendi Buatu, Diamani

\section{Mémoires}

Babineau, Louis

Beaulieu, Caroline

Boulet, Gérard

Carrier, Luc

Castellanos Rodriguez,

Anna Mercedes

Cormier, Françoise

Deschênes, Marc

Fortier, Louis

Fortin, Dominique

Fournier, Bernard

Hufty, Marc

Lacharité, Marcel

Lacourcière, Michel

Laperrère, Johanne
Environnement et changement politique: le multipartisme sénégalais.

L'intégration régionale et sa pertinence pour la self-reliance: le cas de la CEAO.

Les États-Unis et les minéraux stratégiques d'Afrique australe.

Les rapports nippo-américains: esquisse d'une approche psycho-culturelle.

Propositions en vue d'une analyse phénoménologique et structurale.

L'institutionnalisation de l'Assemblée Nationale du Québec: une étude diachronique de la professionnalisation du personnel politique québécois, 1867-1987.

Les relations entre l'état et les firmes multinationales: le cas du Vénézuela 1973-1979.

Une tentative de restructuration de la politique extérieure: le cas du Chili sous le gouvernement Frei (1964-1970).

Le jeu politique des journalistes du pupitre.

Le Canada et la sécurité du Pacifique (de 1970 à aujourd'hui).

L'État de la régulation.

Mouvements de jeunes et socialisation politique: la dynamique de la J.E.C. à l'époque de Gérard Pelletier.

Étude portant sur la relation entre le programme économique et la légitimation du régime militaire argentin de 1976-1981.

La paix non déclarée israélo-arabe.

Canadian Development Assistance to Kenya and Cameroon.

L'influence des impératifs de Radio-Canada, Télémédia et Radiomutuel sur la production radiophonique de leurs stations de base de la région de Québec. 
Le Hir, Françoise

Liu, Kui-Lan

Michaud, Jacinthe

Morin, Gilles

Pacheco-de Roussan, Ana

Pageau, Gilles

Rehayem, Mondher

Rivard, Pierre

Trotier, François

\section{Université de Montréal}

\section{Thèses}

Dalpé, Robert

\section{Larochelle, Gilbert}

Nadeau, Richard

Ouimer, Gérard

Rocher, François

Salée, Daniel
Pratiques journalistiques et action syndicale, au Québec.

Continuités et ruptures dans la politique étrangère chinoise depuis 1979.

L'état des rapports entre le mouvement syndical et les groupes de base issus des nouveaux mouvements sociaux.

Le rôle des journalistes dans la négociation des secteurs public et parapublic.

La dissidence parlementaire au sein des partis politiques québécois (1960-1985).

L'autonomie du directeur général des élections du Québec vue à travers les législations électorales de 1945 à 1985 .

La Ligue arabe et la question palestinienne de 1945 à 1964

Michel Foucault et le bio-pouvoir: pour une approche politiste d'un concept d'économie des modes de vie.

Analyse des contraintes administratives et constitutionnelles de la politique commerciale internationale canadienne.

Innovation et politique technologique dans une petite économie industrialisée ouverte n'exerçant pas le leadership technologique - le cas canadien.

L'imaginaire technocratique: la Commission Trilatérale et sa définition d'un nouvel être ensemble.

Modélisation et analyse empirique des fluctuations de court terme de comportement électoral: le cas canadien.

Érude de la stratification selon les sexes en Europe de l'Est au début des années quatrevingt.

Rapports de pouvoir et intégration économique continentale - 1960-1985: pour une analyse des rapports entre la situation de dépendance, la classe dominante et l'État au Canada.

Changement social et formation de l'État: éléments pour une socio-genèse de l'État canadien (1760-1867). 


\section{Mémoires}

Amar, Daniel

Balladier, Jean-Louis

Beaubien, Michel

Beaulieu, Marc-André

Bédard, Serge

Bernard, Chantal

Blais, Martin

Briand, Marielle

Brosseau, André

Brouillet, Louise

David, Raymond

De Cadaran, Yves Dufour, Ghislain

Diry, Jean

Engulu, Lokanga

Gaurhier, Daniel

Gongora, Thierry

Hansen, Daniel

Irwin, David

Labonté, Benoît

Lalague, François

Lanoville, Carole

Lavoie, Jocelyn

Lepage, Jean-Yves
Les facteurs stratégiques de positionnement en marketing électoral : clarification conceptuelle et nouvelles variables.

Autoritarismes et démocratisations en Amérique latine: les régimes militaires boliviens de 1964 à 1978 .

Les relations Québec-Amérique latine: continuité ou rupture?

L'intervention publique dans l'industrie des équipements électriques au Québec.

Pierre-Elliott Trudeau et la centralisation des pouvoirs politiques stratégiques.

Dépendance et clientélisme; le cas du bassin arachidier sénégalais.

Contenu de la socialisation et changement social.

L'agriculture algérienne 1962-1978: développement? dépendance?

La position de l'industrie québécoise des pâtes et papiers vis-à-vis le libre échange.

Analyse du discours référendaire dans le Devoir du 20 décembre au 20 mai 1980.

Discussion de l'apport théorique des auteurs marxistes contemporains et l'analyse de l'état de droit.

Essai d'interprétation de la pensée de Raymond Aron sur le nationalisme (1940-1977).

Le marketing des comportements. (travail dirigé)

Les dimensions politiques du langage: de Saussure à Hannah Arendt et Noam Chomsky (travail dirigé)

L'exécutif collégial en Uruguay (1952-1966).

Démocratie et décentralisation dans le système politique zaïrois.

Les relations Québec-Belgique

La diplomatie navale coercitive: étude de son emploi par l'URSS et les USA dans le Tiers Monde de 1967 à 1984 .

Dépendance et politique étrangère: le cas du Sultanat d'Oman de 1970 à 1982

La dérèglementation du transport interurbain par autocar au Québec: une recherche portant sur les probables impacts économiques et sociaux.

La déclaration universelle des droits de l'homme et son rayonnement sur les constitutions contemporaines.

État et enjeux des relations France/Irak.

Théorie et pratique du pouvoir du Parti féministe du Canada et du Parti féministe unifié. IBM : un acteur dans le système mondial.

Les politiques gouvernementales du Québec à l'égard des investissements étrangers (1976- 
Mathioudakis, Georgios

Meridji, Tayeb

Michalowski, Tomasz

Noreau, Pierre

Rousseau, Édouard

Tozawa, Fumiko

Turgeon, Yves

Villon, Pierre

\section{Université d'Ottàwa}

\section{Mémoires}

Douab, Ouafa

Drolet, Michael

Harper, Diane

Laferrière, Hubert

Larue, Stéphan

Laver, Patrick

Lavoie, Roland

Ledermann, Robert

LEDUC, Daniel

O'Brien, Gary

Régimbald, André

Tétreault, Daniel

Van Roggen, Trish
Essai sur le "Particularisme" grec en rapport avec le développement/sous-développement et la division internationale du travail.

Les élites franco-manitobaines, 1968-1987.

Bilan cumulatif et comparatif des interprétations théoriques et phénomène du développement économique du Canada.

Le mouvement jeunesse au Québec: niveau d'évolution.

Les objectifs et les effets de l'aide américaine et multilatérale à Haïti: 1971-1982.

L'image et le comportement politique de la femme japonaise contemporaine (après la $2^{\text {ime }}$ guerre mondiale).

La loi des relations ouvrières de 1944.

Théorie et pratique du leadership charismatique. Le cas de Moammar Kadhafi.

Les négociations visant la mise en œuvre de la communauté économique de l'Afrique de l'ouest (CEAO).

Individu, société, Érat chez Rawls: la question des libertés.

Canadian Foreign Aid and the Commonwealth Caribbean: Issues of Local Control.

L'utopie chez $\mathrm{H}$. Marcuse

Analyse comparative de la politique étrangère des pays de la Southern African Development Coordination Conference (SADCC).

Quantitative International Politics and the Foreign Policy Maker.

Image du Canada véhiculée dans la presse d'affaires américaines: revue des méthodes d'analyse des images nationales et contribution à l'étude du cas canadien.

Évaluation et estimation de la fiabilité de sondages électoraux, provinciaux, bipartisans et québécois de la période 1977-1985.

Les barrières non-tarifaires américaines et le déclin commercial et industriel des ÉtatsUnis.

Politique éducationnelle et accumulation du capital: le cas de l'État québécois depuis la "révolution tranquille».

Théorie empirique de connaissance et théorie politique chez John Locke.

L'autonomie politique des autochtones du Canada.

A Test of Dependency Theory - A Case Study of the Ivory Coast. 
Viens, Jacques

\section{Université du Québec à Montréal}

\section{Mémoires}

Debellefeuille, Alain

Leblanc, Marie-France

Vaillancourt, Germaine

Ghernati, Najib

Seghezzo, Anna-Maria

Langlois, Denis

Laliberté, Robert

Tano, Bruno N'gbra

Diallo, Amadou

Belhadj, Fathi

Lavoie, Francine

Lapierre, Jacques

Carre, Eddy

Arcand, Diane

Maaouni, Abdelhak

Thales, Max-Raymond

\section{Université McGill}

\section{Thèses}

Rejali, Darius M.
Les concepts élémentaires de la reconstruction de la théorie du matérialisme historique par Jurgen Habermas.

Dimensions militaires d'Israël.

Les assemblées représentatives du midi de la France au bas moyen âge.

Les québécoises et la santé mentale: de l'élaboration du dispositif psychiarriqque à l'émergence contemporaine de la parole des femmes.

Problèmes du "dialogue istaélo-palestinien» et perspectives de paix au Proche-Orient: 1967-1987.

Nicatagua: Une démocratie participative.

Le déclin du PCF ou une page d'histoire à tourner.

L'imaginaire politique de Victor Segalen.

Le rôle du Québec dans les activités de coopération entre le Canada et l'Afrique francophone (1973-1983).

Le système de recrutement et de sélection de la fonciton publique québécoise de 1943 à 1984, chez les fonctionnaires professionnels.

Problématique de la culture populaire: le cas de la blague politique.

Le développement des politiques scientifiques au Québec.

Le virage technologiques: un virage politique.

État, firmes multinationales et dépendance technologique au Mexique.

Le contrôle des armements négocié entre l'est et l'ouest: le mouvement "pacifiste" ouest européen.

Transfert de technologie et développement: Le cas du Maroc.

Les objectifs du déploiement des euromissiles américains. 
Arel, Dominique

Caisse, Getrude

Costantini, Sandra

De Sousa, Norberto

Degenstein, Paul Richard

Elk, Robert Eugene

Flahault, Michel

Fyles, Stewart R. (Research Paper)

Halewood, Peter

Harvey, Frank

Hoffman, Tod Eric

Iton, Richard

Laferrière, Eric

Lévesque, Michel

Lugar, Jay Andrew

Mayer, Esther

Massand, Sami Gabriel

Michelin, Robert

Molnar, Donald

Morrison, Bruce William

Ritchie, Elizabeth

Sutley, Stewart
Alexander Solghenitoyn's Thought: A Critical Appraisal. (Research paper).

The Electorial System: Its Impact On Feelings Of Alienation (Research Paper).

The Office Of The Commissioner Of Official Languages: An Evaluation (Research Paper).

The Implications Of Annales Historiography For Political Theory (Research Paper).

Western Canadian "Alienation» An Analysis Incorporating David Schwartz's Theory of

Political Alienation (Research Paper).

A Study Of The Effects Of The Southeast Asian Intrusive Power System On The Foreign Policy of Indonesia.

Britain And The Falkland Islands Crisis, 1982: An Analysis of Crisis Decision-Making. The Political Dimensions Of Third World Immigration To Canada: The Case Of Haitian Montreal.

A Critique Of The State And State Power In Clausewitz's On War (Research Paper).

Nuclear Deterrence: An Alternative Approach to Testing (Research Paper).

Recurring Crises In Africa: A Theory Of Continuously Hostile Relationships.

Political Ideology And The Black American Community.

Egyptian Civil-Military Relations (Research Paper).

Le Conservatisme Au Québec: Le Cheminement Politique d'Émilien Lafrance (19521970).

Moral Behevior, Security Imperatives And Bureaucratic Politics: Jimmy Carter's Human Rights Foreign Policy.

Arms Transfers As A Form Of Influence: The Case Of An American-Israeli Patron Client Relationship.

Arab nationalism in the Mirror of Orientalism: A Critique (Research Paper).

Towards A Paradigm In International Relations: Three Perspectives On The Concepts of Kuhn (Research Paper).

The Winnipeg General Strike: Class, Ethnicity, And Class Formation In Canada.

Green Politics in Western Europe: The Distincriveness Of The West German Case, As Compared To Great Britain, France And the Netherlands (Research Paper).

The West German Greens as a New Social Movement (Research Paper).

Losing A Revolution: The PKI Versus The Army In Indonesia 1949-1965. 